\title{
Resonance States of Atomic Anions
}

\author{
ALEXEI V. SERGEEV, SABRE KAIS \\ Department of Chemistry, Purdue University, 1393 Brown Building, West Lafayette, Indiana 47907
}

Received 11 April 2000; revised 29 January 2001; accepted 1 February 2001

\begin{abstract}
We study destabilization of an atom in its ground state with decrease of its nuclear charge. By analytic continuation from bound to resonance states, we obtain complex energies of unstable atomic anions with nuclear charge that is less than the minimum "critical" charge necessary to bind $N$ electrons. We use an extrapolating scheme with a simple model potential for the electron, which is loosely bound outside the atomic core. Results for $\mathrm{O}^{2-}$ and $\mathrm{S}^{2-}$ are in good agreement with earlier estimates. Alternatively, we use the Hylleraas basis variational technique with three complex nonlinear parameters to find accurately the energy of two-electron atoms as the nuclear charge decreases.

Results are used to check the less accurate one-electron model.

(C) 2001 John Wiley \& Sons, Inc. Int J Quantum Chem 82: 255-261, 2001
\end{abstract}

Key words: resonance states; negative ions; complex rotation method; screened Coulomb potential; ionization energy

\section{Introduction}

$\mathbf{T}$ here has long been an interest in the existence of long-lived doubly charged negative atomic ions [1-4]. The possibility of doubly charged negative ion resonances has been raised in the case of oxygen by experiments of Peart et al. [5] who observed resonance-like structures in electron-impact detachment cross section at energies of 19.5 and $26.5 \mathrm{eV}$. A Hartree-Fock calculation of the closedshell electronic configuration shows that the resonance energy of $\mathrm{O}^{2-}$ is about $8 \mathrm{eV}$ above $\mathrm{O}^{-}$, which

Correspondence to: S. Kais; e-mail: kais@power1.chem. purdue.edu.

Contract grant sponsor: Office of Naval Research.

Contract grant number: N00014-97-1-0192.

Contract grant sponsor: NSF Early-Career Award. later was confirmed and modified by configuration interaction and other methods [2]. Sommerfeld et al. [6] performed a large-scale multireference configuration interaction calculation ion using the complex rotation technique to investigate resonance states of $\mathrm{H}^{2-}$. Their results predict the existence of a $\left(2 p^{3}\right)^{4} S$ resonance state of $\mathrm{H}^{2-}$ with a resonance position of about $1.4 \mathrm{eV}$ above the $\left(2 p^{2}\right)^{3} P$ state of $\mathrm{H}^{-}$and a lifetime of $3.8 \times 10^{-16}$ [7], which was confirmed later by complex coordinate rotation calculations of Bylicky and Nicolaides [8]. Recently, the multireference configuration interaction calculations using the complex absorbing potential were performed for $\mathrm{O}^{2-}, \mathrm{S}^{2-}, \mathrm{B}^{2-}$, and $\mathrm{Al}^{2-}$ [9]. No experimental evidence of existence of dianions was found, probably due to insensitivity of scattering processes to dianionic resonances. But, in principle, these resonances could be observed in some scattering experiments [9]. 
In this work, we consider an $N$-electron atom in its ground electronic configuration state with the nuclear charge $Z$ as a variable. Integer values of $Z$ with $Z>N$ correspond to positive ions, with $Z<N$ to negative ions and with $Z=N$ to neutral atoms, where $N$ is the number of electrons. A complex energy of a doubly charged negative ion $(Z=N-2)$ is found by extrapolating the ionization energy into the instability region of nuclear charges less than some critical nuclear charge $Z_{c}$. We use the reliable data for the ionization energy of a neutral atom $(Z=N)$ and its isoelectronic negative ion $(Z=$ $N-1)$, which were calculated or experimentally measured.

The simplest extrapolations by polynomial fits $[10,11]$ or by analytic formulas with a few fitting parameters $[12,13]$ give the real negative binding energy for closed-shell anions such as $\mathrm{O}^{2-}$ or $\mathrm{S}^{2-}$. Extrapolating formula of Herrick and Stillinger [10] includes a singular $3 / 2$ power term and gives both positions and widths of the dianions. Although it correctly models a similar singularity of the energy calculated by a variational method, it fails for the exact energy, which has a less trivial singularity [14].

In our approach, we use the one-electron model to approximate movement of a weakly bound electron that is going to escape when the charge approaches the critical charge. Free parameters of the model are fit in order to reproduce correctly the binding energies of a neutral atom and its isoelectronic anion. Since the existence of one loosely bound electron is a realistic approximation in the vicinity of the critical charge, we believe that the model realistically reproduces the nontrivial singularity at $Z=Z_{c}$ [15]. Earlier, this model was used to determine the critical charges by finding zeroes of the binding energy [16]. It was found that the critical charges never drop below $N-3 / 2$. The maximal surcharge defined as $S_{\mathrm{e}}=N-Z_{\mathrm{c}}$ was found for $\mathrm{Rn}$ isoelectronic series and is equal to 1.48 . Here, we extrapolate the ionization energy deeper into the instability region, to $Z=N-2$, corresponding to a di-anion.

Although various model interaction potentials for a scattering state of $Z+2$ electrons can realistically describe a process of electron-impact detachment from a negative ion (see [17] and references therein), applicability of model potentials for a resonant state of $Z+2$ electrons remains to be studied. There is a common belief that due to a strong correlation effect among several equivalent electrons the underlying physics is much more complicated than a simple model could suggest, and full-scale calcu- lations with multielectron trial wave functions are unavoidable. For $\mathrm{H}^{2-}$, there were studies of potential curves in the framework of hyperspherical coordinates [18] and studies of the interaction between the $N$ th electron and the $(N-1)$-electron target including exchange interaction [19].

In this work, we attempt for the first time to model an unstable multielectron dianion by a suitable one-electron potential. The underlying assumption and the mapping of an atomic system with a weakly bound electron to a one-particle system with a screened Coulomb potential is discussed in the next section. In Section 3, we find an approximate dependence of the energy on the nuclear charge by extrapolation from the known energies of a neutral atom and the corresponding negative ion. Energies and widths of doubly negative ions for isoelectronic series exhibiting a bound singly charged anion are evaluated systematically for $N \leq 18$. Accuracy of the model is estimated by comparison of the model results with exact numerical results at $Z<Z_{c}$ for helium isoelectronic series. Section 4 is a summary and conclusion.

\section{Description of One-Electron Model}

A variational study of the helium isoelectronic series using the Chandrasekhar trial wave function $e^{-a_{1} r_{1}-a_{2} r_{2}}-e^{-a_{1} r_{2}-a_{2} r_{1}}$ [20] shows that $a_{1} / a_{2} \leq 2$ if $Z \geq 2$, where $a_{2}$ is the smallest among the two variational parameters $a_{1}$ and $a_{2}$, in a reasonable agreement that both electrons occupy equivalent orbitals in helium and two-electron positive ions. The ratio of the exponents increases as the nuclear charge decreases and reaches 3.7 for a negative hydrogen ion $(Z=1)$. It means that the orbital of one of electrons inflates in comparison with the orbital of another electron.

A similar variational study of the beryllium isoelectronic series using the function $e^{-a_{1} r_{1}-a_{2} r_{2}-a_{3} r_{3}-a_{4} r_{4}}$ properly symmetrized over coordinates and spin functions shows that $a_{3}=a_{4}$ for beryllium and four-electron positive ions, but $a_{3} / a_{4}=2.4$ for the negative ion of lithium where $a_{3}$ and $a_{4}$ are the smallest exponents corresponding to outer electrons, and $a_{3} \geq a_{4}\left(a_{3} \neq a_{4}\right.$ if $\left.Z \leq 3.25\right)$. Variational study of the neon isoelectronic series by Herrick and Stillinger [10] shows a similar diffusivity of the outer orbital for $\mathrm{F}^{-}(Z=9)$ and for charges slightly less than $9\left(0.111<Z^{-1} \leq 0.118\right)$.

We expect that for $N$-electron atom in general, the outer electronic shell undergoes a profound 
change when the charge decreases and reaches values around $N-1$, so that for a negative ion one of the electrons is held much farther from the nucleus than the others. We admit that some exceptions from this rule are possible. Consider, for example, the $\mathrm{H}^{2-}$ resonance studied in $[7,8]$. It has $\left(2 p^{3}\right)^{4} S$ electronic configuration corresponding to the excited state of the isoelectronic $\mathrm{Li}$. In this system, three electrons occupy three different $p$ orbitals. Since these orbitals are spatially separated along three different axes, three electrons are relatively weakly interacting between themselves. In fact, no electron is pushed out to a loosely bound orbital, so all electrons are equivalent. But at least for atoms in the ground state with an even number of electrons, the last two electrons occupy the same spatial orbital, and we expect that they become unequivalent when the electron repulsion becomes sufficiently strong in comparison with the attraction to the nucleus.

For a given atom with $N$ electrons and a nuclear charge $Z$, let us consider a spherically symmetric potential (also known as Hellmann potential [21]) of the form

$$
V(r)=-\frac{1}{r}+\frac{\gamma}{r}\left(1-e^{-\delta r}\right)
$$

with $\gamma=(N-1) / Z$.

To extrapolate the ionization energy, we suggest here a one-particle model of the outer electron responsible for ionization process moving in an effective potential of the atomic core comprising of the nucleus and $N-1$ electrons. In scaled coordinates $r \rightarrow \mathrm{Zr}$, this potential is approximated by our model potential, Eq. (2.1). Our approximation is asymptotically correct both at small and at large distances from the nucleus where the scaled atomic core potential tends to $-1 / r$ and to $-(Z-N+1) /(Z r)$, respectively. The transition between the two different asymptotic regimes occurs at distances roughly equal to $1 / \delta$, which is about the atomic core radius.

The second parameter of the model potential, Eq. (2.1), $\delta$, is chosen so that the ionization energy in the potential (2.1) is equal to the scaled ionization energy $Z^{-2} E_{\mathrm{I}}(Z)$ of the atom. For atoms with more than two electrons, we consider here an excited state in the potential (2.1) with the same spherical quantum numbers $(n, l)$ as quantum numbers of the loosely bound electron on an external atomic shell (in this aspect our approach differs from the method of pseudo-potentials [22] that deals with the ground state in a potential with an additional repulsive term necessary to satisfy orthogonality conditions). In this way, we map an arbitrary atom, which is characterized by a pair of numbers $(N, Z)$ to the model one-particle system (2.1), which is characterized by a pair of parameters $(\gamma, \delta)$. Results of fitting the parameter $\delta$ for elements with $N \leq 10$ in our previous study [16] give evidence that $\delta$ depends on $1 / Z$ almost linearly.

In summary, our model (2.1) effectively eliminates the singularity in the energy function $E_{\mathrm{I}}(Z)$ to be extrapolated by replacing it with a weakly varying function $\delta(Z)$ that can be accurately extrapolated by a linear dependence on $1 / Z$ without taking into account a complex singularity at $Z=Z_{\mathrm{c}}$. In our previous study [16], we fitted the parameter $\delta$ to meet the known binding energy of the neutral atom and its isoelectronic negative ion and then found $\delta$ as a function of $1 / Z$ by a linear extrapolation. After that, we solved the Schrödinger equation with the potential (2.1) and found some kind of extrapolation of the ionization energy of an atom to the range of $Z<N-1$. Finally, by locating a zero of the ionization energy, we found critical charges for most of atoms with $N \leq 86$. In the present work, we use the same technique to find resonances of doubly charged negative ions by calculating the ionization energy at $Z=N-2$.

\section{Results}

For two-electron isoelectronic series, the parameter $\delta$ is 1.066 and 0.881 for $\mathrm{He}$ and $\mathrm{H}^{-}$, respectively. Decreasing of $\delta$ for $\mathrm{H}^{-}$means increasing of the core radius that is induced by stronger repulsion between electrons. Results of extrapolating the ionization energy to the range $Z<1$ with $\delta$ assumed as a linear function of $1 / Z$ are shown in Figure 1.

To verify our approximation, we performed more accurate variational calculations using the Hylleraas trial wave function of the form

$$
\begin{aligned}
\psi_{N}= & \sum_{i+j+k \leq N} C_{i, j, k}\left[r_{1}^{i} r_{2}^{j} \exp \left(-a r_{1}-b r_{2}\right)\right. \\
& \left.\quad+r_{2}^{i} r_{1}^{j} \exp \left(-a r_{2}-b r_{1}\right)\right] r_{12}^{k} \exp \left(-c r_{12}\right) .
\end{aligned}
$$

First, we found the energy of the negative ion of hydrogen $(Z=1)$ by minimization of the energy functional with respect to three nonlinear parameters $a, b, c$, and the coefficients $C_{i, j, k}$. In agreement with our model of unequivalent electrons, the exponents $a$ and $b$ typically differ by a factor of two or three. For smaller charges $(Z<1)$, the energy was determined by finding a stationary point of the energy functional instead of its minimum. At each step, the charge was decremented by a small amount $\sim 10^{-3}$, and new coordinates of the stationary point 


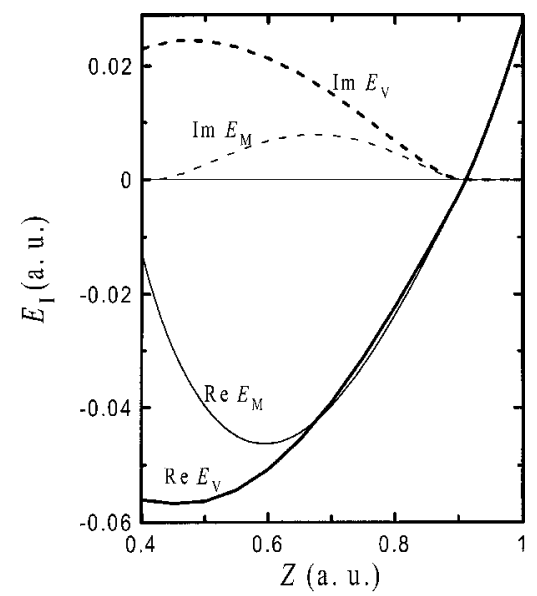

FIGURE 1. Ionization energy for the helium isoelectronic series as a function of the nuclear charge including an instability region $Z<Z_{c}$. Bold lines are accurate results found by the variational method with a large basis set. Thin lines are results of the simplified one-particle model described in Section 2. Solid and dashed lines are real and imaginary parts, respectively.

were calculated by an iteration procedure taking coordinates of the stationary point from the previous step as an initial guess to search a changed stationary point (for slightly decreased charge). In this way, we found that the optimized parameters $a, b$, and $c$ are real for sufficiently large charges. If the charge is lower than some value, then the minimum of the energy functional disappears and turns to a complex stationary point (this feature is typical for any system passing from a bound to a quasi-stationary state that is treated variationally, for example, for $\mathrm{Ne}$ isoelectronic series with a nuclear charge below $Z_{c}=8.74$ [10]). We found numerically the parameters $a, b, c$, and corresponding energy in the range $0 \leq Z \leq 1$ with $N$ up to 5 .

The above method is a more general version of the complex rotation or the complex stabilization method [23]. Instead of one nonlinear complex variational parameter, the rotation angle, we are using three nonlinear variational parameters $a, b$, and $c$. Junker [24] used a similar variant of the complex stabilization method with several nonlinear variational parameters. Like the method of Junker [24], our method gives correct resonance energies without taking into account an asymptotical form of the resonant wave function, or imposing specific constrains on the parameters $a, b$, and $c$.

We used the complex parameters $a, b$, and $c$ calculated for the particular case of $N=5$ in order to extend calculations to higher $N$ by optimizing only the linear coefficients $C_{i, j, k}$. Almost exact" variational energy calculated at $N=25$ differs from the variational energy at $N=5$ in the amount of less than $10^{-4}$. It is compared in Figure 1 with results of one-electron model calculations. The real part of the ionization energy is always negative at $0<Z<Z_{c}$. It reaches its minimum at $Z \approx 0.5$, and the width reaches its maximum approximately at the same point. For small $Z$, convergence of the variational method becomes worse. It seems that both real and imaginary parts tend to zero when $Z \rightarrow 0$.

As expected, the one-electron model gives fairly accurate results in the vicinity of the critical charge where it models the threshold singularity. Here, the real part is approximated more accurately than the imaginary part because the singularity manifests mostly in the imaginary part.

Dubau and Ivanov [14] calculated the twoelectron atom resonance in the vicinity of the critical charge using $1 / Z$ expansion and the complex rotation method. Their results agree with our calculation; see Table I. Since in the latter case the charge is close to the critical charge, we slightly corrected location of the singularity in our model by fitting $\delta$ at points $Z=Z_{\mathrm{c}}=0.911028225$ and $Z=2$ instead of fitting it at points $Z=1$ and $Z=2$.

For isoelectronic series of $\mathrm{Li}$ in its ground state $\left(1 s^{2} 2 s\right)^{2} S$, our model cannot be used because $\mathrm{He}^{-}$ does not exist in the $\left(1 s^{2} 2 s\right)^{2} S$ state. However, in the excited $\left(2 p^{3}\right)^{4} S$ state the negative ion $\mathrm{He}^{-}$is bound nonrelativistically. We tried to use our model for this excited state by taking the ionization energies as a difference between the energies of $\left(2 p^{3}\right)^{4} S$ states listed in [25] and the energies of $\left(2 p^{2}\right)^{3} P$ states with the same $Z$ listed in [26]. The parameter $\delta$ is 0.239 and 0.204 for $\mathrm{Li}$ and $\mathrm{He}^{-}$, respectively. Extrapolation of the ionization energy to the range $Z<2$ by the method described in Section 2 shows that $E_{\mathrm{I}}(Z)$ is zero at $Z=1.92$, and it has minimum at $Z \approx 1.3$, while the width has maximum at

TABLE I

Resonances in the helium isoelectronic series for charges below the critical charge.

\begin{tabular}{ccccc}
\hline $1 / Z$ & $-Z^{-2} \operatorname{Re} E$ & $-Z^{-2} \operatorname{Im} E$ & $\begin{array}{c}-Z^{-2} \operatorname{Im} E \\
\text { (CRM [14]) }\end{array}$ & $\begin{array}{c}-Z^{-2} \operatorname{Im} E \\
\text { (model) }\end{array}$ \\
\hline 1.11 & 0.497131 & 0.000050 & 0.00006 & $\sim 0.00003$ \\
1.12 & 0.494953 & 0.000286 & 0.00028 & 0.00024 \\
1.13 & 0.492792 & 0.000686 & 0.00070 & 0.00057 \\
1.14 & 0.490616 & 0.001207 & 0.00121 & 0.00098 \\
\hline
\end{tabular}


$\mathrm{Z} \approx 1.4$. For $\mathrm{H}^{2-}$, our extrapolation gives $E_{\mathrm{I}}(1)=$ $-0.029+0.00002 i$ (a.u.). Our values for the position of the resonance $-\operatorname{Re} E_{I}$ and its half-width $\operatorname{Im} E_{\mathrm{I}}$ are considerably lower than results of complex rotation calculations [8], $E_{\mathrm{I}}=-0.053+0.031 i$. It is likely that our extrapolation becomes poorer as the charge drops below the point where the extrapolation has a minimum, as it can be seen on Figure 1 for heliumlike ions. A simple threepoint quadratic extrapolation gives $E_{\mathrm{I}}(1) \approx 3 E_{\mathrm{I}}(2)-$ $3 E_{\mathrm{I}}(3)+E_{\mathrm{I}}(4)=-0.0298$, which is very close to the prediction of our model, while an extrapolation with use of Eldén's [12] three-parameter formula does not work because it has a nearby pole at $Z \approx 1.3$. It seems that any extrapolation over the parameter $1 / Z$ from stable to unstable anions is unreliable for light atoms because $1 / Z$ differs considerably for singly and doubly negative ions if $N$ is small.

For four-electron isoelectronic series, results of using our model for extrapolating the ionization energy are shown in Figure 2. We found that the curve hits the border of continuum spectrum $E_{\mathrm{I}}=0$ at $Z_{\mathrm{c}}=2.864$ [16] and again at $Z_{\mathrm{c}}^{\prime}=2.17$. Very small imaginary part exists in the interval $Z_{\mathrm{c}}^{\prime}<Z<Z_{\mathrm{c}}$. The extrapolation gives positive ionization energy for $\mathrm{He}^{2-}, E_{\mathrm{I}}=0.064$. Since a bound state of $\mathrm{He}^{2-}$ does not exist, it means that we deal with a virtual state with an exponentially growing wave function (corresponding to a pole of the scattering function on the second sheet of the Riemann surface). It may be also possible that because of its limitations our

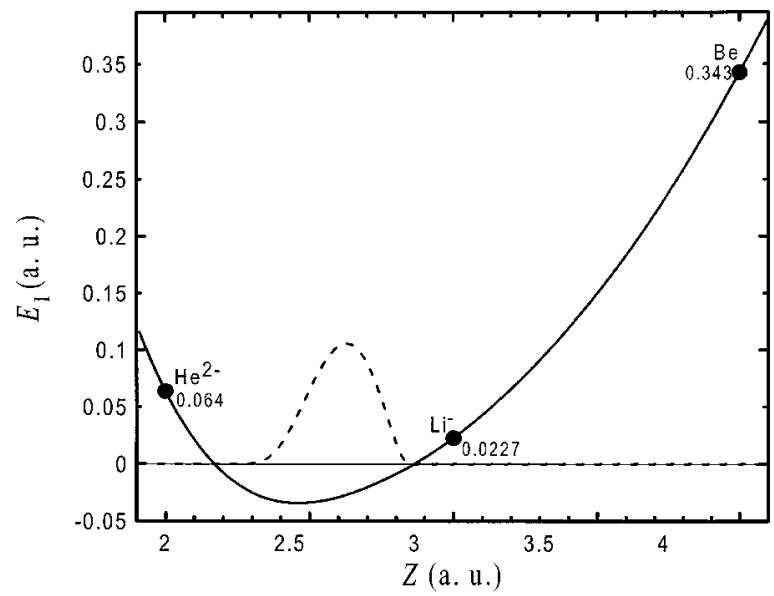

FIGURE 2. Ionization energy for the beryllium isoelectronic series extrapolated from bound systems $\mathrm{Be}$ and $\mathrm{Li}^{-}$to a resonance state of $\mathrm{He}^{2-}$. A dashed line is an imaginary part of the energy times 100 . The system $\mathrm{He}^{2-}$ with a zero imaginary part is presumably in a virtual state.

method fails for the $\mathrm{He}^{2-}$ ion. We found positive ionization energies for another doubly charged negative ions of noble gases, for example, $\mathrm{Ne}^{2-}$ (see Table III).

The doubly charged negative ion of atomic oxygen in the same electronic configuration as a closedshell 10-electron configuration of a neutral atom of neon was extensively studied theoretically. Comparison of the ionization energy of $\mathrm{O}^{2-}$ found by different methods is given in Table II. For critical

TABLE II

Energy of $\mathrm{O}^{2-}$ resonance calculated by various methods.

\begin{tabular}{llc}
\hline \multicolumn{1}{c}{ Reference } & \multicolumn{1}{c}{ Method } & $-E_{\mathrm{I}}(\mathrm{eV})$ \\
\hline Clementi and McLean [27] & Hartree-Fock & 8.30 \\
Huzinaga and Hart-Davis [28] & Hartree-Fock-Roothaan ${ }^{\mathrm{a}}$ & 7.68 \\
Cantor [29] & Combining thermochemical data & 7.94 \\
Herrick and Stillinger [10] & Extrapolating formula including 3/2 power term & $5.38-0.65 i$ \\
& Eldén's [12] three-parameter formula & 5.31 \\
& Kaufman's [13] two-parameter formula & 7.17 \\
& Kaufman's [13] three-parameter formula & 6.53 \\
Gadzuk and Clark [30] & Quadratic fit & 7.09 \\
Robicheaux et al. [11] & Extrapolating of isoelectronic cases & 8.8 \\
Sommerfeld [9] & Polynomial fit & $7.2^{\mathrm{b}}$ \\
Present work & Complex absorbing potential & $5.77-0.87 i$ \\
\end{tabular}

\footnotetext{
${ }^{a}$ Huzinaga and Hart-Davis listed the total energy. We found ionization energy by subtracting the Hartree-Fock total energy of the corresponding singly charged negative ion that is listed in works of Clementi et al. [27, 31].

${ }^{\mathrm{b}}$ Estimated width is greater than $1 \mathrm{eV}$.
} 


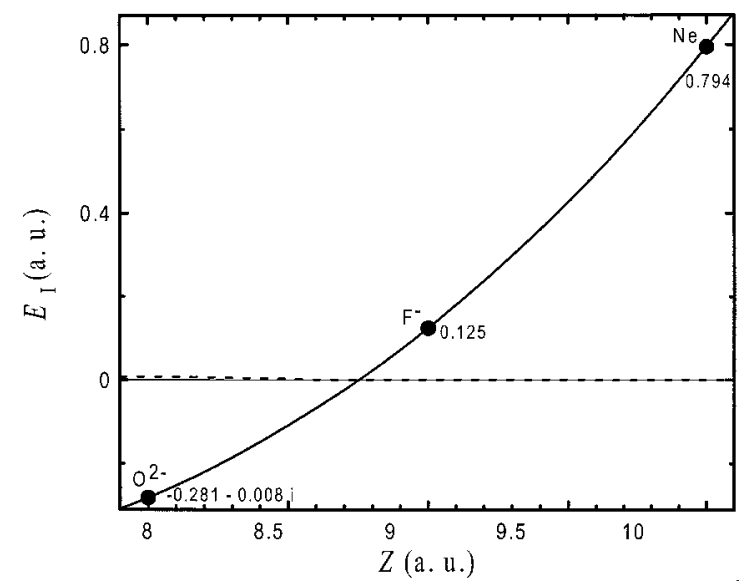

FIGURE 3. Ionization energy for neon isoelectronic series extrapolated from bound systems $\mathrm{Ne}^{2}$ and $\mathrm{F}^{-}$to a resonance state of $\mathrm{O}^{2-}$. A dashed line is an imaginary part of the energy, which is half-width of the level.

discussion of some of these results, see [10]. Our result for the resonance position is in agreement with most of the above results. However, our prediction of the resonance width is significantly lower than that of Herrick. It may be attributed to the fact that Herrick's 3/2-power term, which is responsible for the width, is far from the actual singular behavior or to the fact that our model systematically underestimates the width as it happens for two-electron atoms. Recent multireference configuration interaction calculations of Sommerfeld [9] predict still larger width. However, this prediction may be only an upper bound to the true resonance width because of possible missing correlation effects that stabilize the metastable state [9]. The extrapolated ionization energy as a function of $Z$ is shown in Figure 3. It has a relatively small nonlinear distortion and a weak singularity at $Z_{c}=8.75$.

We extended calculations of resonances of doubly charged negative ions to the first two rows of the periodic table. Here, we considered only the ions for which an isoelectronic singly charged negative ion is stable, for example, we omitted $\mathrm{C}^{2-}$ because $\mathrm{N}^{-}$ does not exist. Results are listed in Table III. For the closed-shell ion $\mathrm{S}^{2-}$, our result for the resonance position $-\operatorname{Re} E_{\mathrm{I}}$ is somewhat larger than the previous estimates.

Herrick and Stillinger [10] suggested that stability of negative ions increase with decreasing of $\gamma=$ $(N-1) / Z$, or the effective charge seen asymptotically by the outer electron. A greater stability would then be expected for the $S^{2-}$ ion $(\gamma=1.0625)$ than for the $\mathrm{O}^{2-}$ ion (1.125). Our results for half-width of
TABLE III Energies of doubly charged negative ions. ${ }^{a}$

\begin{tabular}{ccc}
\hline$Z$ & $-E_{I}(\mathrm{eV})$ & Lifetime $\left(10^{-14} \mathrm{~s}\right)$ \\
\hline $2 \mathrm{He}^{2-}$ & -1.73 & \\
$4 \mathrm{Be}^{2-}$ & 3.43 & 1.1 \\
$5 \mathrm{~B}^{2-}$ & 4.88 & 0.3 \\
& $3.95^{\mathrm{b}}$ & $0.036^{\mathrm{b}}$ \\
$6 \mathrm{C}^{2-}$ & $6.11^{\mathrm{c}}$ & \\
$7 \mathrm{~N}^{2-}$ & 7.48 & 0.12 \\
& $7.97^{\mathrm{d}}$ & \\
& $7.07^{\mathrm{c}}$ & \\
$10 \mathrm{Ne}^{2-}$ & -0.92 & \\
$12 \mathrm{Mg}^{2-}$ & 1.79 & 3.0 \\
$13 \mathrm{Al}^{2-}$ & 3.44 & $0.067^{\mathrm{b}}$ \\
& $3.02^{\mathrm{b}}$ & 0.5 \\
$14 \mathrm{Si}^{2-}$ & 4.76 & \\
& $4.12^{\mathrm{c}}$ & 0.5 \\
$15 \mathrm{P}^{2-}$ & 4.95 & 0.7 \\
& $4.47^{\mathrm{c}}$ & $0.071^{\mathrm{b}}$ \\
$16 \mathrm{~S}^{2-}$ & 4.91 & \\
& $3.90^{\mathrm{b}}$ & \\
& $4.62^{\mathrm{c}}$ & \\
& $4.7^{\mathrm{e}}$ & \\
\hline
\end{tabular}

a Tabulated energies are results of the present work unless marked otherwise. For energy of $\mathrm{O}^{2-}$ ion, see Table II.

${ }^{b}$ Complex absorbing potential method of Sommerfeld [9].

${ }^{c}$ Hartree-Fock-Roothaan calculations of Huzinaga and HartDavis [28].

d Hartree-Fock calculations of Clementi and McLean [27].

e Polynomial fit of Robicheaux et al. [11].

$\mathrm{S}^{2-}$ and $\mathrm{O}^{2-}, 0.045$ and $0.22 \mathrm{eV}$, respectively, as well as results of Sommerfeld [9] (0.45 and $0.87 \mathrm{eV}$ ) support this argument. However, the widths for $\mathrm{B}^{2-}$, $\mathrm{Al}^{2-}, \mathrm{S}^{2-}$ calculated by Sommerfeld [9] are larger than our results by one order in magnitude.

\section{Discussion}

We have investigated the existence of resonance states for doubly charged atomic negative ions by considering the behavior of the ground state with a decrease of the nuclear charge. When the charge drops below the critical charge, the bound state goes into a complex plane and becomes a resonance. We attempt to approximate reasonably accurate the function $E_{\mathrm{I}}(Z)$ in the vicinity of the critical charge $Z_{\mathrm{c}}$ where it exhibits a singularity. Approximating of isoelectronic ionization potentials by simple analytic formulas with several adjustable constants was 
widely used before appearance of powerful computers more than 40 years ago. However, these formulas seem inadequate for negative ionization potentials, which are complex-valued, with an imaginary part meaning a half-width. Herrick and Stillinger [10] introduced a singular term $\sim\left(Z-Z_{c}\right)^{3 / 2}$ to their extrapolating scheme to approximate the complex energy at $Z<Z_{c}$.

Our method is some kind of refinements of Herrick and Stillinger method of analytic continuation. We believe that our model reproduces the threshold singularity of the energy more realistically than the 3/2-power singularity of Herrick and Stillinger because it is based on a physical model that is realistic at $Z \approx Z_{\mathrm{c}}$. Generally, the method of analytic continuation from bound to resonance states appears very accurate when the threshold behavior of the energy is incorporated into continuation scheme, as it was demonstrated earlier for two-particle resonances in nuclear physics; see Chapter 5 of Ref. [32].

The developed method was checked for a twoelectron atom, for which the Hylleraas basis variational technique gives reliable results. Position and width of the resonance were compared with a prediction of the less accurate model approximation. Results were found in agreement.

The results of our approximate one-electron model agree with earlier estimates of closed-shell resonances of $\mathrm{O}^{2-}$ and $\mathrm{S}^{2-}$. In addition, the model predicts similar resonances other dianions that are isoelectronic to some bound singly charged anion.

Our model invites further generalizations, for example, by considering a motion of two weakly bound electrons instead of only one.

Although no multiple negative ions exist in a bound state [16, 33, 34], some long-lived resonances of doubly negative ions are observed experimentally [1]. Our results give evidence for some resonances that remain to be observed.

\section{ACKNOWLEDGMENTS}

We would like to acknowledge the financial support of the Office of Naval Research (N00014-97-10192). One of us (S.K.) acknowledges support of an NSF Early-Career Award.

\section{References}

1. Buckman, S. J.; Clark, C. W. Rev Mod Phys 1994, 66, 539.

2. Bates, D. R. Adv Atom Mol Opt Phys 1991, 27, 1.

3. Kalcher, J.; Sax, A. F. Chem Rev 1994, 94, 2291.

4. Scheller, M. K.; Compton, R. N.; Cederbaum, L. S. Science 1995, 270, 1160

5. Peart, B.; Forrest, R. A.; Dolder, K. T. J Phys B 1979, 12, 847.

6. Sommerfeld, T.; Riss, U. V.; Meyer, H.-D.; Cederbaum, L. S. Phys Rev Lett 1996, 77, 470.

7. Sommerfeld, T.; Riss, U. V.; Meyer, H.-D.; Cederbaum, L. S. Phys Rev A 1997, 55, 1903.

8. Bylicki, M.; Nikolaides, C. J Phys B At Mol Opt Phys 1998, 31, L685.

9. Sommerfeld, T. Phys Rev Lett 2000, 85, 956.

10. Herrick, D. R.; Stillinger, F. H. J Chem Phys 1975, 62, 4360.

11. Robicheaux, F.; Wood, R. P.; Greene, C. H. Phys Rev A 1994, $49,1866$.

12. Eldén, B. J Chem Phys 1960, 33, 98.

13. Kaufman, M. Astrophys J 1963, 137, 1296.

14. Dubau, J.; Ivanov, I. A. J Phys B At Mol Opt Phys 1998, 31, 3335.

15. Ivanov, I. A. Phys Rev A 1995, 52, 1942.

16. Sergeev, A. V.; Kais, S. Int J Quantum Chem Symp 1999, 75, 533.

17. Pedersen, H. B.; Djuricét, N. Phys Rev A 1997, 55, 1903.

18. Morishita, T.; Lin, C. D. Phys Rev Lett 1998, 80, 464.

19. Chung, K. T. Phys Rev A 1998, 58, 2777.

20. Rau, A. R. P. J Astrophys Astr 1996, 17, 113.

21. Hellmann, B. J Chem Phys 1935, 3, 61.

22. Callaway, J. Phys Rev 1958, 112, 322.

23. Ho, Y. K. Phys Repts 1983, 99, 1.

24. Junker, B. R. Phys Rev Lett 1980, 44, 1487.

25. Davis, B. F.; Chung, K. T. Phys Rev A 1990, 42, 5121.

26. Midtal, J. Phys Rev 1965, 138, A1010.

27. Clementi, E.; McLean, A. D. Phys Rev 1964, 133, A419.

28. Huzinaga, S.; Hart-Davis, A. Phys Rev A 1973, 8, 1734.

29. Cantor, S. J Chem Phys 1973, 59, 5189.

30. Gadzuk, J. W.; Clark, C. W. J Chem Phys 1989, 91, 3174.

31. Clementi, E.; McLean, A. D.; Raimondi, D. L.; Yoshimine, M. Phys Rev 1964, 133, A1274.

32. Kukulin, V. I.; Krasnopol'sky, V. M.; Horáček, J. Theory of Resonances; Kluwer: Dordrecht, 1989.

33. Kalcher, J. Chem Phys 1987, 115, 33.

34. Hogreve, H. J Phys B At Mol Opt Phys 1998, 31, L439. 